\title{
Emi1 stably binds and inhibits the anaphase-promoting complex/cyclosome as a pseudosubstrate inhibitor
}

\author{
Julie J. Miller, ${ }^{1}$ Matthew K. Summers, ${ }^{3}$ David V. Hansen, ${ }^{2}$ Maxence V. Nachury, ${ }^{3}$ \\ Norman L. Lehman, ${ }^{3}$ Alex Loktev, ${ }^{3}$ and Peter K. Jackson ${ }^{2,3,4,5,6}$ \\ ${ }^{1}$ Program in Molecular Pharmacology, Stanford University School of Medicine, Stanford, California 94305, USA; ${ }^{2}$ Program in \\ Cancer Biology, Stanford University School of Medicine, Stanford, California 94305, USA; ${ }^{3}$ Department of Pathology, \\ ${ }^{4}$ Department of Microbiology and Immunology, Stanford University School of Medicine, Stanford, California 94305, USA; \\ ${ }^{5}$ Genentech, Inc., San Francisco, California 94080, USA
}

\begin{abstract}
The periodic destruction of mitotic cyclins is triggered by the activation of the anaphase-promoting complex/cyclosome (APC/C) in mitosis. Although the ability of the APC/C to recognize destruction box (D-box) substrates oscillates throughout the cell cycle, the mechanism regulating APC/C binding to D-box substrates remains unclear. Here, we show that the APC/C inhibitor Emi1 tightly binds both the APC/C and its Cdh1 activator, binds to the D-box receptor site on the $\mathrm{APC} / \mathrm{C}^{\mathrm{Cdh} 1}$, and competes with $\mathrm{APC} / \mathrm{C}$ substrates for D-box binding. Emi1 itself contains a conserved C-terminal D-box, which provides APC/C-binding affinity, and a conserved zinc-binding region (ZBR), which antagonizes APC/C E3 ligase activity independent of tight APC binding. Mutation of the ZBR converts Emi1 into a D-box-dependent APC/C substrate. The identification of a direct Emi1-APC/C complex further explains how Emi1 functions as a stabilizing factor for cyclin accumulation and the need to destroy Emi1 for APC/C activation in mitosis. The combination of a degron/E3 recognition site and an anti-ligase function in Emi1 suggests a general model for how E3 substrates evolve to become pseudosubstrate inhibitors.
\end{abstract}

[Keywords: Mitosis; maturation-promoting factor; ubiquitin-protein ligases; Polo-like kinases; anaphase-promoting complex]

Supplemental material is available at http://www.genesdev.org.

Received May 31, 2006; revised version accepted July 14, 2006.

Periodic accumulation and destruction of cyclins is essential for the oscillation of cyclin-dependent kinase activity that allows progression through the cell cycle. The anaphase-promoting complex or cyclosome (APC/C), an E3 ubiquitin ligase, is responsible for the timely proteasome-dependent destruction of cyclins, as well as other key substrates essential for cell cycle regulation (Harper et al. 2002). The APC/C is a multicomponent complex, composed of at least 10 subunits in mammals, including a Cullin homolog APC2 and a RING-H2 finger protein APC11 (Yu et al. 1998; Zachariae et al. 1998), which are at the core of the essential ubiquitin chain-forming activity.

Interestingly, the abundance of core $\mathrm{APC} / \mathrm{C}$ subunits does not oscillate throughout the cell cycle, but APC/C substrate recognition activity is stimulated in mitosis (Harper et al. 2002; Yamano et al. 2004). The timing and

${ }^{6}$ Corresponding author.

E-MAIL pjackson@gene.com; FAX (650) 225-4000.

Article published online ahead of print. Article and publication date are online at http://www.genesdev.org/cgi/doi/10.1101/gad.1454006. regulation of APC/C activation in mitosis is not fully understood but certainly reflects a balance of positive and negative factors. APC/C activation requires the association of the APC/C with a WD40-repeat-containing activator protein. To date, two major activator proteins have been identified, Cdc20 and Cdh1 (Schwab et al. 1997; Visintin et al. 1997). The mechanism by which these proteins activate the APC/C is not fully understood. APC/C substrates are recognized through conserved degrons, either the destruction box (D-box) RxxL (Glotzer et al. 1991; King et al. 1996) or an alternative degron, the KEN box (Pfleger and Kirschner 2000). One site for recognition resides within the Cdc20 and Cdh1 activators (Pfleger et al. 2001). Specifically, APC ${ }^{C d c 20}$ is thought to recognize substrates containing D-boxes, whereas $\mathrm{APC}^{\mathrm{Cdh} 1}$ appears to have the additional capacity to bind substrates containing KEN boxes. Recent studies support that Cdc20 binds specifically to the D-box and Cdh1 to both KEN and D-boxes (Burton and Solomon 2000; Hilioti et al. 2001; Pfleger et al. 2001). A recent analysis, however, suggested that D-box peptides also bind directly to the core APC/C (Yamano et al. 2004), 
raising the possibility that the core APC/C contains a D-box receptor. While this finding suggested a primary interaction of D-box with the APC/C core, it does not exclude a multistep mechanism in which D-boxes interact with APC/C activators and with the APC/C core at distinct times during the ubiquitination process. The study by Burton et al. (2005) detected an association between the D-box/KEN box substrate Hsll and TAP-purified APC/C only in the presence of Cdh1. This study and another by Kraft et al. (2005) suggest that specific sequences on the WD40 domain of Cdh1 are required for direct D-box binding. Several models may reconcile these differences, including the possibility that there may be D-box-binding sites on both WD40 activators and the APC/C core itself. Indeed, the APC/C subunit APC10/Doc1 has been implicated as a D-box receptor, and evidence suggests it is critical for bridging the interaction between D-box and KEN-box substrates and

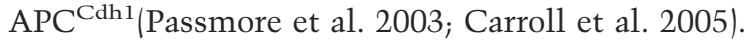

To date, a number of inhibitors of the APC/C have been identified, including components of the spindle checkpoint (Hoyt et al. 1991; Li and Murray 1991; Castro et al. 2005; Nasmyth 2005), the APC/C inhibitor Emi1 (Reimann et al. 2001a,b), and the recently described RASSF1A (Jackson 2004; Song et al. 2004). The role of the spindle assembly checkpoint, notably the Cdc20binding proteins Mad2 and BubR1, is important to restrain the activation of the APC/C and the destruction of the chromosome cohesion regulator securin at the metaphase-anaphase transition in vivo (Castro et al. 2005; Nasmyth 2005). Mad2 and the other components of the spindle checkpoint (Mad1, Bub1, BubR1, Mps1) do not appear important for the difference between interphase and mitotic levels of APC/C activity, but instead are implicated in the timing of events in mitosis /den Elzen and Pines 2001; Geley et al. 2001; Meraldi et al. 2004).

The primary inhibitor of the APC/C during interphase is the protein Emi1, which has been shown to be required for the accumulation of cyclins in HeLa cells (Hsu et al. 2002), for entry into mitosis in Xenopus embryos (Reimann et al. 2001a), and also to efficiently inhibit the APC/C in vitro (Reimann et al. 2001b). A homolog of Emi1, Emi2/Erp1, also directly inhibits the APC/C in meiosis (Schmidt et al. 2005; Tung et al. 2005). Previous studies showed that Emil could associate with the APC/C activators Cdc20 and Cdh1 (Reimann et al. 2001a,b; Hsu et al. 2002). Moreover, the mitotic destruction of Emil triggered by the $\mathrm{SCF}^{\mathrm{BTrCP}}$ ubiquitin ligase was shown to be required for the activation of cyclin A and cyclin B destruction (Guardavaccaro et al. 2003; Margottin-Goguet et al. 2003). Finally, the requirement of the polo kinase Plk1 for the mitotic destruction of Emil (Hansen et al. 2004; Moshe et al. 2004) and for full activation of the APC/C, suggests the model that polo kinases could affect APC/C activation by triggering the destruction of an interphase APC/C inhibitor.

Emil did not appear to be an APC/C substrate itself but instead required an extrinsic, SCF-dependent pathway for its destruction. Emil destruction is tightly linked to nuclear envelope breakdown, generally thought to the irreversible step in commitment to mitosis (Rieder and Maiato 2004) and a prerequisite for APC/C activation. Emil is thus also a strong candidate for the factor that provides a critical lag between activation of the mitosis-promoting activity (MPF) of cyclin B/Cdc2 and activation of the APC/C that causes cyclin B destruction. This lag is important for preventing premature destruction of accumulating cyclin, to allow full activation of MPF. Emil's role in controlling APC/C activity in early mitosis suggests it may participate in a recently described prometaphase timer (Meraldi et al. 2004).

To better understand how Emil inhibits cyclin destruction in interphase, we purified Emil-associated proteins from interphase HeLa cells and found that Emil associates in a complex with the APC/C and Cdh1. Further, we find that Emil can bind to the $\mathrm{APC} / \mathrm{C}$ via a conserved C-terminal destruction box and can compete for APC/C-substrate interaction. The ability of Emil to inhibit the APC/C derives from a combination of its conserved D-box, which provides strong APC/C-binding affinity and a block to substrate-APC/C binding, and a conserved zinc-binding region (ZBR), which provides an APC/C E3 ligase antagonizing activity. We find that the ZBR works by blocking substrate access to the APC/C, but through a possible steric mechanism independent of D-box binding. Inactivating this ZBR function turns Emil from an APC/C inhibitor into an APC/C substrate, supporting the idea that Emil functions as a pseudosubstrate inhibitor of the APC/C to allow cyclin accumulation.

\section{Results}

Emil associates with the nuclear form of the APC

To better understand the mechanism of Emil control of the APC/C in interphase, we purified Emil-associated proteins from interphase HeLa cells. Low salt and high salt extracts were made from asynchronous (mostly interphase) cells and examined by immunoblotting for Emil and the APC/C subunits APC3/Cde27 and APC8 (Fig. 1A). The majority of these proteins were extracted from the cell pellets after $0.42 \mathrm{M} \mathrm{KCl}$ extraction. Both Emil and APC/C are largely nuclear in interphase, which may require a nuclear or chromatin anchor.

To examine whether Emil and the APC/C are directly associated, we separated the extracted protein complexes on a $\mathrm{Q}_{\text {sepharose }}$ anion exchange $(\mathrm{Q})$ column and observed coelution of Emi1, Cdc27, and the activator protein Cdh1 at $\sim 420 \mathrm{mM} \mathrm{KCl}$. Other APC/C subunits coeluted similarly (Supplementary Fig. 1A). This 0.42-M KCl fraction was then resolved by gel filtration and the APC/C subunit APC3/Cde27 coeluted with Emi1, in a broad complex of $\sim 1 \mathrm{MDa}$. We have not yet clearly determined any specific subcomplexes.

Sizing of the extracted Emil and APC/C directly on gel filtration chromatography showed cofractionation of these proteins in the $1 \mathrm{MDa}$ complex (Supplementary Fig. 1B), although a more substantial fraction of the 
Figure 1. Emil associates with the nuclear form of the APC. (A) Emil and the APC/C are coextracted from the high-salt fraction of HeLa cells. HeLa cells were extracted by sequential treatments of nitrogen decompression, $420 \mathrm{mM}$ $\mathrm{KCl}, 0.1 \%$ Triton $\mathrm{X}-100$, and sonication to produce fractions as described in Materials and Methods. Fractions were examined by immunoblot analysis for Emil and APC/C subunits (APC3/Cdc27, APC8). (B) Emil, Cdh1, and $\mathrm{APC} / \mathrm{C}$ cofractionate during ion exchange and gel filtration chromatography. The nuclear fraction described in $A$ was further fractionated by anion exchange (upper panel) chromatography and subsequently separated by gel filtration (lower panel). (C) Emil and APC/C coprecipitate from purified fractions. The fractions indicated in $B$ were pooled and immunoprecipitated with anti-Emil antibodies and resolved by SDSPAGE and silver stained. Excised bands were identified by mass spectrometry. APC/C subunits identified by mass spectromety are indicated by arrows. $(D)$ Anti-Emil antibody immunoprecipitates were also examined by immunoblot analysis for Emil and APC/C subunits, APC3 (Cdc27), APC5, and APC7. (E) AntiAPC3/Cdc27 efficiently coprecipitates Emi1 from nuclear fractions. Immunoprecipitates were washed with $0.1,0.25$, or $0.5 \mathrm{M} \mathrm{KCl}$; separated by SDS-PAGE; and examined by immunoblot analysis for Cdc27 and Emil. (F) APC/C subunits associate with expressed epitope-tagged Emil. HeLa cells were transfected with a construct expressing epitope-tagged Myc-hEmil or empty vector. Cell extracts were prepared and anti-Myc precipitates were examined by immunoblot analysis for the presence of Emil and APC/C subunits. (G) Immunodepletion of asynchronous nuclear extract by anti-Emil antibody results in the removal of the majority of APC/C and Cdh1. Nuclear extracts were incubated with anti-Emil or control antibody. Equal amounts of input and flow-through (nonbound) samples were examined by immunoblotting.

Emi1-APC/C complex appears in a high-molecular-mass complex of at least $\sim 3 \mathrm{MDa}$. The physical behavior of this larger complex appears to reflect the association of Emil-APC/C with additional factors (K. Ban, J.J. Miller, and P.K. Jackson, unpubl.). Separation of HeLa extract by sucrose gradient also revealed cosedimentation of Emil and $\mathrm{APC} / \mathrm{C}$ in a large complex of $\sim 1 \mathrm{MDa}$ (Supplementary Fig. 1C).

There are two visible forms of the Emil protein (p51 and p49|, consistently seen with either affinity-purified rabbit anti-Emil antibodies or with a specific Emil monoclonal antibody (Fig. 1B). The lower mobility form appears selectively associated with the APC in either the fractionated $\sim 1-\mathrm{MDa}$ complex (Fig. 1B) or in the highmolecular-mass complex (Supplementary Fig. 1B). Additional fractionation by anion exchange chromatography appears to separate Emil and the APC/C from a largermolecular-weight complex, but we have not fully determined the composition of this larger complex. The consistent cofractionation of Emil and the APC/C following extraction, ion exchange, and gel filtration suggested that Emil and the APC/C remain specifically and tightly associated under a variety of conditions.

To characterize which proteins are associated with Emi1, we immunoprecipitated Emil from the 0.42-M $\mathrm{KCl} \mathrm{Q}$ fraction and examined the identity of associated proteins by mass spectrometry (Fig. 1C) and by immunoblotting for candidate Emil-associated proteins (Fig. 1D). Specific gel regions centered at 210, 100, and $75 \mathrm{kDa}$ were excised, analyzed by mass spectrometry, and identified as APC/C subunits APC1, APC3/Cdc27, and APC6/Cdc16. We performed immunoblots of the Emil coimmunoprecipitated material for other APC/C subunits, and found that $\mathrm{APC} 3 / \mathrm{Cdc} 27, \mathrm{APC} 5$, and $\mathrm{APC} 7$ (Fig. 1D), and APC1, APC4, APC6/Cdc16, APC8/Cdc23, and APC 11 (data not shown) were specifically associated with the complex. As further validation, Emil interacted with both Cdh1 and APC/C in a reconstitution of purified Emil with the APC/C (see Fig. 2).

We also observed Emil-APC/C association when we immunoprecipitated APC3/Cdc27 from the high-salt-extracted HeLa fraction (Fig. 1E), demonstrating that the association can be seen by precipitation of either partner. Of interest, the association between Emil and the $\mathrm{APC} / \mathrm{C}$ was stable in $0.25 \mathrm{M} \mathrm{KCl}$ but was partially disrupted in $0.5 \mathrm{M} \mathrm{KCl}$, suggesting that the Emil-APC/C interaction is more labile than interactions among the core subunits of the APC/C. A previous study noted that Emil can interact efficiently with the activator protein Cdc20 but did not detect an interaction between Emi1 and APC/C in Xenopus extracts (Reimann et al. 2001a). We suspect the Emil-APC/C interaction was over- 
A

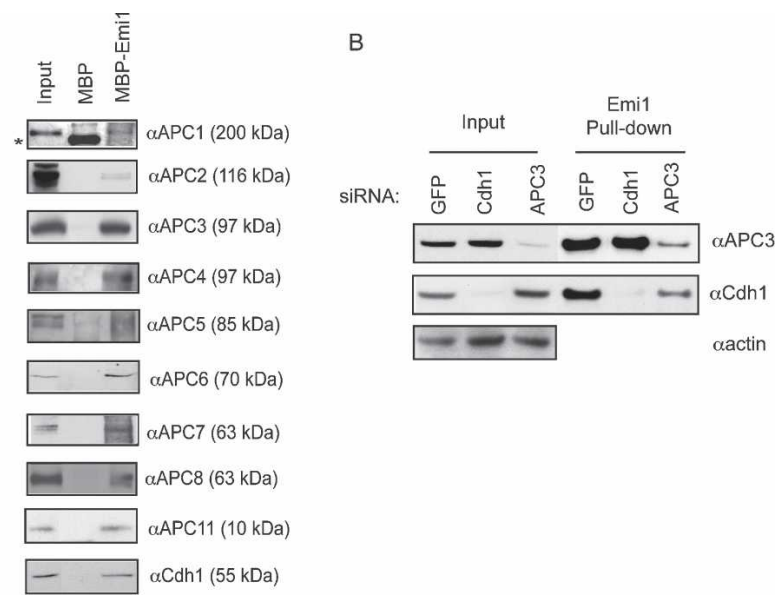

Figure 2. Immobilized Emil can bind to the $\mathrm{APC} / \mathrm{C}^{\mathrm{Cdh} 1}$ in an "APC/C capture" assay. (A) Recombinant Emil protein immobilized on Sepharose beads (see Materials and Methods) was incubated with HeLa cell extracts, and precipitates were analyzed for APC/C binding by immunoblot analysis. The approximate molecular weight at which the indicated subunit migrates is indicated in parentheses. (B) Emil binds to the APC/C in the absence of Cdh1. Emil beads were incubated with extracts made from HeLa cells transfected with siRNA targeting Cdh1 or APC3 for $48 \mathrm{~h}$.

looked because of competition with the more prevalent Emi2/Erp1 protein, the meiosis-specific homolog of Emil (Schmidt et al. 2005; Tung et al. 2005).

As an additional validation of Emil-APC/C interaction, we expressed epitope-tagged Emil in human embryonic kidney 293T cells and found efficient coprecipitation of the APC3/Cdc27 protein (Fig. 1F). We were able to deplete the majority $(>80 \%)$ of Emil from the extract using an anti-Emil antibody and found that Cdh1 $(>80 \%)$ and the APC/C were efficiently codepleted (Fig. 1G), suggesting that a majority of Cdh1 and the APC/C are associated with Emil during interphase.

\section{The APC/C binds to immobilized Emi1} in a 'capture' assay

Emil efficiently inhibits the APC ${ }^{\text {Cdh1 } 1}$ in vitro (Reimann et al. 2001b) and in vivo (Hsu et al. 2002). Here, the identification of an Emil-APC/C complex suggests that Emil may inhibit the core APC/C directly. To test the mechanism of the Emil-APC/C interaction, we used an in vitro binding or "capture" assay to reconstitute and study this interaction. This assay is similar to that used by Yamano et al. (2004) to study cyclin B-APC/C association. Purified, recombinant Emil was immobilized on Sepharose beads, and these beads were incubated with HeLa cell lysates to allow binding of the APC/C. The bound complex was then assayed by immunoblotting. As shown in Figure 2A, a full panel of known APC/C subunits, including the Cdh1 activator, was found to be associated with Emil.

Previous reports had noted an interaction between
Emil and the APC/C activators Cdh1 and Cdc20 (Reimann et al. 2001a,b; Hsu et al. 2002). We wondered if the interaction between Emil and the APC/C was strictly through this previously described association between Emil and activators, or if Emil is able to bind directly to the APC/C core, independently of Cdc20 and Cdh1. We tested whether loss of Cdh1 by RNA interference disrupts the Emil-APC/C interaction in asynchronous HeLa cells. We found that we could efficiently deplete levels of Cdh1 in HeLa cells using small interfering RNA (siRNA) and, further, we found that Cdc20 does not detectably associate with the APC/C in asynchronous extracts (Supplementary Fig. 1I). Here, we observed that Emil could capture APC/C with equal efficiency in both the presence and absence of Cdh1 (Fig. 2B).

Yamano et al. (2004) found that the ability of the cyclin B D-box to bind to the APC/C occurred in Xenopus egg extracts depleted of Cdc20, which are absent of appreciable Cdh1. We also found that Emil could efficiently capture the APC/C in Xenopus egg extracts depleted of Cdc20 (Supplementary Fig. 1G). Thus Emil, much like cyclin B, can capture the APC/C independent of activators Cdc20 and Cdh1.

To further characterize the interaction between Emi1 and $\mathrm{APC} / \mathrm{C}$, we targeted a number of $\mathrm{APC} / \mathrm{C}$ subunits with siRNA. Overall, Emil capture of the APC/C was acutely sensitive to perturbations in the abundance of certain subunits. Depletion of APC3/Cdc27 significantly reduced the amount of APC/C captured by Emil, as detected by immunoblotting with antibodies recognizing a number of other subunits (Fig. 2B; Supplementary Fig. $1 \mathrm{H})$. Intriguingly, the reduced amount of APC3 also somewhat reduced the amount of Cdh1 captured by Emil, suggesting that the APC/C can influence the Emil-Cdh1 interaction.

Emil binding to the D-box receptor on the APC/C
is more dependent on the D-box than on the ZBR

To define which structural elements in Emil are important for APC/C binding and inhibition, we examined the ability of immobilized Emil variants (Fig. 3A) to bind $\mathrm{APC} / \mathrm{C}$ in the capture assay. We found that the Emil C terminus (amino acids 299-447) efficiently bound to both the APC/C and Cdh1, whereas the N-terminal portion (1-244) of Emil did not bind (Fig. 3B), consistent with earlier results showing the $\mathrm{C}$ terminus as the APC/C inhibitory domain (Reimann et al. 2001a).

The $\mathrm{C}$ terminus of Emil contains a ZBR (see Fig. 3A) conserved in homologs of Emil and Emi2/XErp1 (Schmidt et al. 2005; Tung et al. 2005) and also found in the TRIAD family of E3 ubiquitin ligases, including the E3 ubiquitin ligase parkin (van der Reijden et al. 1999). Examination of the Emil and Emi2/Erp1 primary sequences also showed the presence of conserved destruction boxes in the conserved C-terminal domain (Supplementary Fig. 2A), a region of Emil required for APC/C inhibition. Here, we found that the ability of Emil to capture the APC/C was strongly dependent on its D-box, as Emil protein containing a mutation of the D-box 
Miller et al.

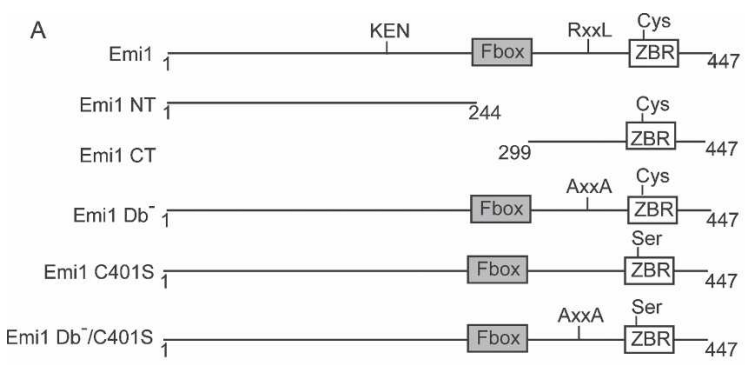

B

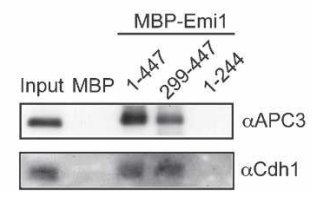

C

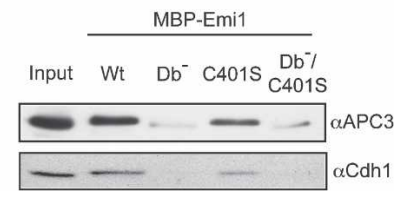

Figure 3. The $\mathrm{APC} / \mathrm{C}$ binds to the $\mathrm{C}$-terminal $\mathrm{APC} / \mathrm{C}$ inhibitory domain of Emil via its conserved D-box. $(A)$ Emil protein schematic, key features, and variant proteins. $(B)$ The Emil Cterminal domain binds to the APC/C and Cdh1. Capture of the APC/C using recombinant MBP-Emil N terminus (1-244) or C terminus (299-447). (C) Efficient Emil binding to the APC/C and $\mathrm{Cdh} 1$ requires the $\mathrm{C}$-terminal D-box. Capture of APC/C using MBP-Emil D-box mutant $\left(\mathrm{Db}^{-}\right)$, MBP-Emil ZBR mutant (C401S), or double mutant $\left(\mathrm{Db}^{-} / \mathrm{C} 401 \mathrm{~S}\right)$.

$\left(\mathrm{R}_{322} \mathrm{xxL} \rightarrow \mathrm{AxxA}\right)$ significantly reduced APC/C binding. Mutation of a conserved cysteine (C401) in the ZBR only reduced APC/C binding slightly. A double mutant with both the D-box and C401S mutations showed minimal binding to the APC/C, similar to the D-box mutant (Fig. 3C). The requirements for the interaction between Emil and Cdh1 were similarly strongly dependent on an intact D-box (Fig. 3C; Supplementary Fig. 2C).

We consistently observed two distinct forms of APC3 interacting differentially with the Emil wild-type and D-box mutant proteins. A faster migrating form of APC3 associates specifically with the Emil D-box mutant. While we do not fully understand the nature of this species, we suspect it may reflect a specific phosphorylation state of the APC/C that gates the Emi1-APC/C interaction.

Previous analyses of Emil showed that ZBR function was critical for APC/C inhibition (Reimann et al. 2001b). However, as we see here, the D-box contributes strongly to Emil-APC/C binding and the ZBR has a modest or minimal contribution to binding, suggesting a distinct mechanism for the ZBR in APC/C inhibition. We were interested in further defining that distinct function.

Emi1 competes with D-box-dependent, APC/C substrate binding to the D-box receptor on the $A P C / C$

Substrate destruction boxes are important for both ubiquitination and binding to the $\mathrm{APC}^{\mathrm{Cdh} 1}$ or $\mathrm{APC}^{\mathrm{Cdc} 20}$ ligases (Burton et al. 2005; Kraft et al. 2005). To extend these analyses, we used cyclin B, a well-validated APC/C substrate, to demonstrate D-box-dependent binding to the APC ${ }^{\mathrm{Cdh} 1}$. First, we validated that an immobilized N terminus of cyclin B efficiently captured the APC/C in HeLa extract (Fig. 4A). In this assay, similar to that in Xenopus extract published by Yamano et al. (2004), the binding of cyclin B to the APC/C is strongly dependent on the D-box.

Because Emil also binds to the D-box receptor, we tested whether Emil or a control protein (MBP) would compete with cyclin B binding to APC ${ }^{\mathrm{Cdh} 1}$. We found that the Emil protein efficiently blocked D-box binding, but that the control protein did not (Fig. 4B). Emil was also able to compete for $\mathrm{APC}^{\mathrm{Cdh} 1}$ binding with securin, another D-box APC/C substrate (Supplementary Fig. 2B). Emil was also able to compete with the interaction between cyclin B and Cdh1 (Supplementary Fig. 2D), similar to a previous report showing that Emil can compete with the ability of securin to interact with Cdc20 (Reimann et al. 2001b). Therefore, Emil protein can bind both Cdh1 and the APC/C and is capable of efficiently blocking both APC/C and Cdh1 capture by D-box substrates.

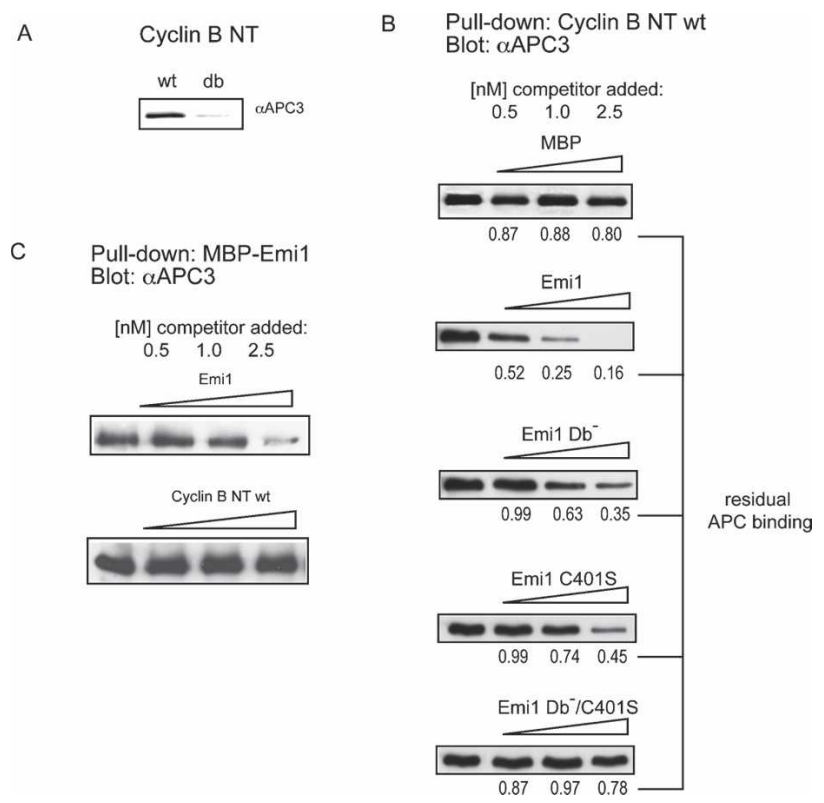

Figure 4. Emil can compete with substrate binding in D-boxand ZBR-dependent manner. $(A)$ The normal $\mathrm{N}$-terminal D-box region of cyclin $\mathrm{B} 1$ binds specifically to the APC/C. Recombinant $\mathrm{N}$ terminus of cyclin B1 (residues 1-100) or a variant containing a mutated D-box was immobilized on CNBr-activated Sepharose, incubated with cell extracts, and analyzed for APC/C binding with anti-Cdc27 antibody. (B) Emil competes with the ability of the cyclin B1 D-box region to bind the APC/ C. Recombinant wild-type or mutant MBP-Emil or MBP $(0.5,1$, or $2.5 \mathrm{nmol}$ ) was added to $1 \mathrm{nmol}$ bead-bound cyclin $\mathrm{B} 1$ and incubated with HeLa cell extracts as described in $A$. The amount of APC/C remaining bound to Cyclin B beads is indicated below, expressed as a percentage of control (no Emil addition) sample. Values represent mean of two experiments. $(C)$ Cyclin B1 does not compete efficiently with Emil for APC/C binding. $\mathrm{N}$ terminus of cyclin B1 or MBP-Emil was added at doses of $0.5,1.0$, or $2.5 \mathrm{nmol}$ to capture assay using $1 \mathrm{nmol}$ of bead-bound MBP-Emi1. 
To examine whether the ability of Emil to compete D-box binding requires its D-box or ZBR, we tested the ability of single D-box $\left(\mathrm{Db}^{-}\right)$or ZBR (C401S) mutants and the double mutant $\left(\mathrm{Db}^{-} / \mathrm{C} 401 \mathrm{~S}\right)$ to compete cyclin $\mathrm{B}$ binding. We found that the D-box mutant and the ZBR mutant are both partially compromised in their ability to compete with cyclin B (Fig. 4B), but that the double mutant does not measurably compete. Although this demonstrates that the ZBR and D-box are each sufficient to inhibit substrate binding, they appear to inhibit by different mechanisms. The ability of the D-box mutant to compete with cyclin B binding to the APC/C suggests that the ZBR prevents substrate access to the APC/C in a D-box-independent manner. An interesting hypothesis is that the Emil ZBR is positioned within the APC/C holoenzyme to sterically exclude substrates from the APC/C D-box receptor and active site. Structural studies will be important to test this hypothesis.

We wanted to test if, as an interphase APC/C inhibitor, Emil was more effective in binding to the APC/C than its natural substrates, like cyclin B. Therefore, we tested the ability of cyclin B N terminus to compete with APC/C capture by immobilized Emil beads. We found that cyclin B competes for Emil binding to the APC/C poorly (Fig. 4C), suggesting that Emil has a significantly higher affinity than a natural substrate.

The results in Figure 4, B and C, also suggest that Emil uses the $\mathrm{ZBR}$ as an additional activity to prevent cyclin B binding. A fragment of Emil containing only the ZBR (amino acids 378-419) retains a modest ability to both capture the APC/C from HeLa extract and compete with cyclin B-APC/C binding (Supplementary Fig. 2E). Taken together, these results suggest the D-box enables Emil to bind efficiently to the APC/C whereas the ZBR can block access of APC/C substrates to the core enzyme. In addition to blocking substrate binding, this inhibition may work by inactivating other functions important for APC/C catalysis, although we have currently excluded
E1-E2 charging and E2-APC/C binding (Supplementary Fig. 2F). Previous work has shown that Emil does not inhibit the activity of APC2/APC11 in a minimal reaction (Reimann et al. 2001b).

\section{Emil's function as an inhibitor of APC/C E3 ligase activity requires contributions from its $D$-box and $Z B R$ motifs}

To further understand the contribution of the Emil Dbox and ZBR to its APC/C inhibitory function, we tested the effect of mutations in these sequences on their ability to inhibit the APC/C using an in vitro ubiquitination assay. Here, we found Emil was able to inhibit the ability of the APC/C to ubiquitinate cyclin B to background levels at a dose of $0.5 \mu \mathrm{M}$ (Fig. 5), consistent with previous reports (Reimann et al. 2001b). The ZBR mutant C401S required higher concentrations of $\sim 4-8 \mu M$ to maximally inhibit APC/C activity. Likewise, mutation of the Emil D-box (RxxL $\rightarrow$ AxxA) also strongly reduced Emil's APC/C inhibitory activity, with concentrations of $8 \mu \mathrm{M}$ now being required to maximally inhibit. A double mutant lacking both D-box and ZBR was unable to inhibit the APC/C, even at concentrations exceeding $8 \mu \mathrm{M}$. Thus, we find that both the D-box and ZBR are required for Emil to efficiently inhibit the APC/C.

In these experiments, the Emil inhibitor was present throughout the ubiquitination experiment. As a more stringent test of Emil's APC/C inhibitory activity, we preincubated recombinant Emil wild-type or mutant versions with immunopurified APC/C for $30 \mathrm{~min}$ and then washed the beads to remove any unbound Emil. Again, the Emil double mutant failed to inhibit APC/C activity, and the single D-box mutant and ZBR mutant both exhibited less activity. The D-box mutant appeared to be a less effective inhibitor in this assay, presumably because of a decreased ability to bind to the APC/C (Supplementary Fig. 3A,B). Thus, although both the ZBR

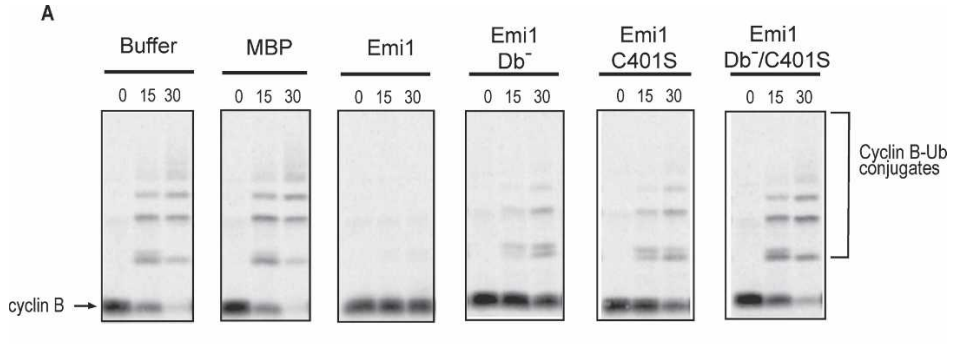

B

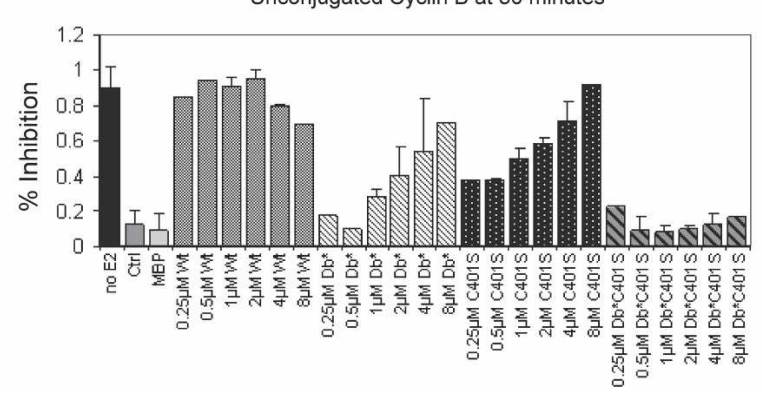

Figure 5. Emil's APC/C inhibitory function requires both the conserved D-box and ZBR functions. (A) APC/ $\mathrm{C}^{\mathrm{Cdh} 1}$-dependent in vitro ubiquitination assays, using radiolabeled, in vitro translated cyclin $\mathrm{B} 1$ protein as a substrate, were incubated for the indicated times $(0,15$, $30 \mathrm{~min})$, and the formation of ubiquitinated cyclin B was visualized by autoradiography of SDS-polyacrylamide gels. Wild-type, D-box mutant $\left(\mathrm{Db}^{-}\right)$, ZBR mutant (C401S), and D-box, ZBR double-mutant Emil was added to the reaction mixture and assayed for the ability to inhibit cyclin B ubiquitination. (B) Quantitation of in vitro APC/C activity showing the amount of cyclin $\mathrm{B}$ remaining unconjugated at $30 \mathrm{~min}$ for a range of doses of Emil variants. 
and D-box can contribute to APC/C inhibitory activity in vitro at higher concentrations than are presumably seen in vivo, Emil requires both D-box-dependent APC/ C-binding function and ZBR-dependent ability to block substrate binding, presumably through an interaction outside the D-box receptor. We believe these interactions reflect the normal in vivo function of wild-type Emil, which may remain associated with specific pools of the $\mathrm{APC} / \mathrm{C}$ in interphase.

Mutation of the Emi1 ZBR converts Emi1 from an $A P C / C$ inhibitor to a $D$-box dependent $A P C / C$ substrate

We found that the conserved D-box in Emil is able to bind to the APC/C D-box receptor, but that the ZBR provided an independent APC/C inhibitory function. We hypothesized that Emil might function as an APC/C substrate in the absence of the ZBR. Therefore, we tested the ability of wild type and the ZBR mutant of Emil to be ubiquitinated by the APC/C. We found that whereas wild-type Emil protein was a poor APC/C substrate, the ZBR mutant was efficiently ubiquitinated (Fig. 6A). Further, we found that the ZBR-D-box double mutant was not efficiently ubiquitinated, compared with the ZBR single mutant. Thus, lacking its essential ZBR-dependent APC/C inhibitory function, Emil becomes an efficient D-box-dependent APC/C substrate. Viewed in this context, by combining a D-box receptor-binding site and an additional ZBR-dependent inhibitory activity, Emil functions as a pseudo-substrate inhibitor of the APC/C.

We also examined whether Emil lacking its ZBR function could serve as a substrate of the APC/C in vivo. Previous studies showed that overexpression of APC/C activators causes a decrease in levels of $\mathrm{APC}^{\mathrm{Cdh} 1}$ substrates (Bashir et al. 2004). We cotransfected the HAtagged APC/C activator Cdh1 in HEK293T cells along with Myc-Emil variants and found that levels of wildtype Myc-Emil protein were slightly reduced by Cdh1 overexpression (Fig. 6B, left panel). This suggests that Emil may have a limited ability to serve as an APC $^{\text {Cdh1 }}$ substrate, or may simply represent a nonspecific effect of cotransfection. However, the levels of the Myc-Emil C401S mutant were strongly reduced by Cdh1 overexpression (Fig. 6B, right panel). In agreement with our in vitro results, the effect of Cdh1 overexpression on the ZBR mutant could be reversed by additionally mutating the D-box. Transfection of a form of Cdh1 containing mutations in APC/C-interacting motifs ( $\triangle$ Cbox, $\Delta \mathrm{IR})$, which have been shown to interfere with Cdh1 activator activity, failed to reduce Myc-Emil C401S levels (Fig. 6B) indicating that the observed changes in MycEmil levels upon Cdh1 overexpression are a result of APC $^{\text {Cdh } 1}$-dependent destruction.

The Cdh1-induced decrease was not a result of the known Emil-SCF ${ }^{\beta T r C P}$ destruction pathway (MargottinGoguet et al. 2003; Hansen et al. 2004), as mutation of the conserved $\beta$ TrCP degron DSGxxS in the Emil sequence (S145/9A) had no effect on the ability of Cdh1 to
A

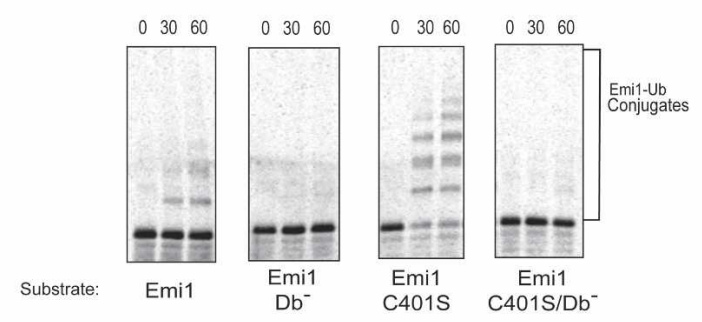

B

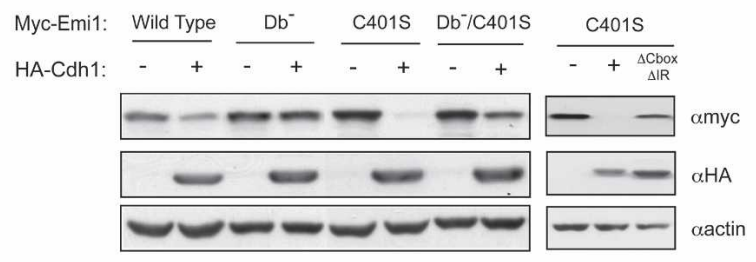

c

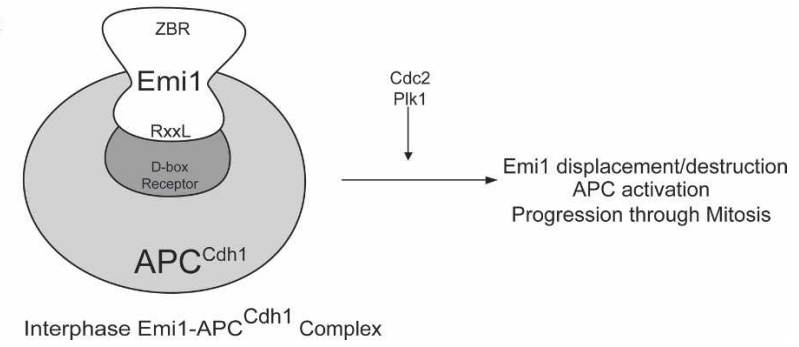

Figure 6. Loss of IBR/ZBR function in Emil converts the protein from APC/C inhibitor to APC/C substrate. (A) Standard APC/C-dependent ubiquitination assays, using radiolabled, in vitro translated C-terminal Emil wild-type or mutant protein as substrate. Reactions were incubated for $60 \mathrm{~min}$, and the formation of ubiquitinated Emil was visualized by autoradiography of SDS-polyacrylamide gels. $\left(\mathrm{Db}^{-}\right)$D-box mutant. $(B)$ Cdh1 overexpression reduces levels of cotransfected Emil C401S/ZBR. HA-Cdh1 and Myc-Emil variants were cotransfected into HEK293T cells. Lysates were harvested and Emil levels detected by anti-Myc immunoblot. A mutant version of Cdh1 lacking the C-terminal IR dipeptide and the C-box ( $\Delta$ Cbox $\Delta \mathrm{IR})$ was used as a control. (C) A model for evolving APC/C substrates and inhibitors. Emil protein binds to the $\mathrm{APC} / \mathrm{C}$ in a D-box-dependent manner to inhibit APC/C activity through G2 and early mitosis, permitting cyclin accumulation. The ZBR motif of Emil contributes to inhibition by blocking substrate access to the APC/C, as well as providing an additional inhibitory function that prevents the ubiquitination of substrates and Emil itself. The combination of D-box and ZBR allows Emil to compete with APC/C substrates for APC/C binding. Phosphorylation of Emil by Cdc2 and Plk1, potentially in conjunction with other events, results in the displacement and proteolytic destruction of Emil, allowing for APC/C activation and progression through later stages of mitosis.

cause a decrease in Myc-Emil levels (Supplementary Fig. 3C).

We observed that the Emil ZBR mutant behaved similarly in Xenopus extracts in which the APC/C is activated by the addition of exogenous Cdh1 (Supplementary Fig. 3D). The bulk of in vitro translated Emil ZBR mu- 
tant was degraded by $30 \mathrm{~min}$, while the ZBR-D-box double mutant remained stable even at $60 \mathrm{~min}$.

\section{Discussion}

The function of Emil as an APC/C inhibitor is clearly important for the S-phase accumulation of cyclins and progression to mitosis, best demonstrated in HeLa cells (Hsu et al. 2002). Here, we find that Emil forms a natural, high-molecular-weight complex with the APC ${ }^{\mathrm{Cdh} 1}$ complex. This complex may account for the majority of the APC/C in the nucleus of interphase cells. Within this context, our data support the idea that the normal function of Emil in interphase cells is to persistently associate with and inhibit the APC/C.

We studied the requirements for Emil binding to the APC/C using an immobilized Emil-binding assay. Here, Emil-APC/C association required a conserved D-box in the Emil $\mathrm{C}$ terminus to provide the majority of the APC ${ }^{\mathrm{Cdh} 1}$-binding affinity, whereas the ZBR had a modest contribution to binding. Either the D-box or the ZBR is sufficient to partially antagonize substrate binding to the APC/C, and each contributes to the ability of Emil to block efficient ubiquitin chain formation. Because the Emil ZBR does not contribute strongly to Emil-APC/C binding but nonetheless retains the ability to block substrate binding to the $\mathrm{APC} / \mathrm{C}$, it appears that the $\mathrm{ZBR}$ does not inhibit through the $\mathrm{D}$-box receptor, but through a separate site on the APC/C. We suggest that the ZBR may be positioned to sterically exclude access to the D-box-binding site on the APC ${ }^{\mathrm{Cdh} 1}$. Once properly oriented on the APC/C using its D-box, the Emil may use the ZBR to act as a plug to block substrates and/or key ubiquitination enzymes from reaching the active site of the APC/C.

Our previous work demonstrated a direct association of Emil with APC/C activators Cdc20 (Reimann et al. 2001a) and Cdh1 (Reimann et al. 2001b; Hsu et al. 2002), which provided one mechanism for APC/C inhibition. The ability of Emil to bind purified Cdh1 directly does require the Emil D-box. The ability of Emil's D-box to associate with the major APC/C complex, including Cdh1, suggests that Emil either binds the APC/C via a D-box receptor on Cdh1, through a separate D-box receptor on the APC/C, or a combination of the two. The possibility of a direct D-box receptor on the APC/C was suggested by Yamano et al. (2004) based on their finding that the cyclin B D-box associated with the APC/C in Xenopus extracts lacking Cdh1 and Cdc20. We similarly found that Emil can bind the APC/C in the absence of Cdc20 and Cdh1 in Xenopus egg extract. However, Burton et al. (2005) have shown that in yeast Cdh1 is required for a D-box substrate Hsll to bind to purified APC/C. It remains possible that an unidentified APC activator, possibly one specific to meiotic cells as found in other organisms, or additional factors in higher organisms may direct or enhance binding to D-box substrates directly with APC/C.

Does Emil bind to the APC only through Cdh1? Interestingly, we find that Emil depletion removes almost
$100 \%$ of Cdh1 from HeLa extracts and at least $80 \%$ of the APC/C. However, the levels of Cdh1 appear to be a small, substoichiometric fraction of the levels of the APC holoenzyme. Further, depletion of Cdh1 from HeLa extract did not affect Emil capture of the APC/C. Thus, it is unlikely that Cdh1 serves as a persistent physical bridge between Emil and the APC/C. We favor the model that Emil interacts first with Cdh1 to be loaded onto the APC/C and that there is an independent D-box receptor, such as APC10/Doc1, on the APC that will allow binding of D-box proteins like Emil and cyclin B. The D-box receptor within the core APC/C revealed by this study and that of Yamano et al. (2004), which both used a high concentration of D-box protein immobilized on beads, is of significantly lower affinity than that of the D-box receptor on the activators. The apparently higher affinity of Emil to Cdh1 than the APC/C may be important given the lower abundance of Cdh1, or may suggest an ordered interaction with Cdh1 first and the APC/C second.

The stable high-affinity association of Emil and the $\mathrm{APC} / \mathrm{C}$ in interphase raises the critical questions of when and how Emil is displaced from the APC/C to relieve inhibition. The destruction of Emil by a SCF ubiquitin ligase is triggered by the Polo-like kinase Plk1 and accelerated by cyclin B/Cdc2 kinase (Hansen et al. 2004; Moshe et al. 2004), leading to the activation of the APC/C in early mitosis (Fig. 6C). Because Emil tightly binds the APC/C core in interphase, the displacement of Emil from the APC/C may be required before it can be targeted for ubiquitin-dependent destruction. This displacement step could be regulated by the same mitotic kinases required for Emil destruction.

Recent studies have shown that a highly conserved homolog of Emi1, called Erp1/Emi2, plays a critical role in inhibiting the APC/C in Meiosis II to prevent activation of cyclin destruction in unfertilized eggs (Schmidt et al. 2005; Tung et al. 2005). Like Emil, Erp1/Emi2 also contains a D-box and ZBR domain within the C-terminal inhibitory domain (Fig. 3A; Supplementary Fig. 2A). We find that Emi2 does associate with the APC/C (J.J. Miller and P.K. Jackson, unpubl.), supporting the idea that Emi2 would also form a complex with the APC/C as part of its APC/C inhibitory function in meiosis II.

The ability of a conserved degradation sequence, or degron, paired with a catalysis-inhibitory function suggests a general model for evolving substrates of ubiquitin ligases into regulatory inhibitors. Similar models for pseudosubstrate inhibition of other regulatory enzymes, notably protein kinases (Russo et al. 1996), suggest that coupling a high-affinity binding site or catalysis inhibitory domain to a typical peptide substrate site provides an efficient enzyme inhibitor. A recent study suggested that the $\mathrm{SCF}^{\beta \mathrm{TrCP}}$ ubiquitin ligase may have a pseudosubstrate regulator as the chromatin-associated hnRNP-U protein may regulate substrate binding (Davis et al. 2002), although a specific $\beta$ TrCP degron within this protein was not identified. The possibility that other pseudosubstrate inhibitors of E3 ubiquitin ligases may couple conserved degrons with specific E3 inhibitory do- 
mains suggests a means for how these inhibitors may be identified.

The ordered destruction of APC/C substrates, notably cyclin A, securin, and cyclin B, ensures that specific steps in mitosis occur in the correct sequence. One way to achieve this ordered timing is by tuning the affinity or Km of each substrate to allow the "best" substrate to be destroyed first and lower-affinity substrates would be destroyed later. Here, the queue of destruction of substrates would be linked to the rank order of $\mathrm{Km}$ values for each substrate. Maximum discrimination of timing is further enhanced when the APC/C is limiting and the rate of substrate destruction is saturated. Here, the "best" substrates serve as inhibitors to push lower-affinity substrates further back in the queue. Additional regulatory events and localization mechanisms may contribute to and expand the relative timing. However, given that the time cells spend in interphase may vary greatly, there must be a mechanism to release the queue at the appropriate time in mitosis. Further, if the "best" substrate is destroyed first in the queue, what prevents this substrate from binding to the APC/C and being destroyed prematurely? By evolving a high-affinity D-box protein like Emil into an inhibitor, the cell can use the single event of destroying the inhibitor-cued by mitotic entry and nuclear envelope breakdown-to initiate the timed and ordered destruction of cyclin A, securin, and cyclin B after the highly variable time spent in interphase.

\section{Materials and methods}

\section{Antibodies}

The following antibodies were used for immunoblotting: rabbit anti-hEmil (Hsu et al. 2002), mouse monoclonal anti-hEmil (raised against full-length MBP-Emil fusion protein), mouse anti-Cdc27 (610454; BD Transduction Laboratories), rabbit antiAPC1, APC2, APC5, APC8 (Hongtao Yu, University of Texas Southwestern Medical Center), rabbit anti-APC4, APC6, APC7, APC11 (Jan-Michael Peters, Research Institute of Molecular Pathology), mouse monoclonal anti-Myc 9E10 antibody (Teresa Wang, Stanford University), rabbit anti-Cdc20 (Edgar Kramer, Max-Planck Institute), rabbit anti-Cdc20 (Zymed), and mouse anti-Cdh1 (NeoMarkers). Rabbit anti-Emi1, rabbit anti-Cdc27 raised against GST-hCdc27, and rabbit anti-myc (sc-789; Santa Cruz Biotechnology/ were used for immunoprecipitation.

\section{Plasmids and purified proteins}

Constructs for wild-type and D-box mutant cyclin B N terminus were obtained from Guowei Fang (Stanford University) and purified by standard methods. pCS2 + Myc-hEmil was previously described (Hansen et al. 2004) and site-directed mutagenesis was performed using QuikChange protocol. pMal-Emil site-directed mutants were created by standard methods and verified by sequencing. Recombinant MBP-Emil fusion proteins were purified as described previously (Hsu et al. 2002). Cdh1 was expressed in Sf9 cells and purified by standard methods.

\section{HeLa cell extraction}

HeLa whole-cell pellet was resuspended in Buffer A / $10 \mathrm{mM}$ Tris at $\mathrm{pH} 7.4,10 \mathrm{mM} \mathrm{KCl}, 1.5 \mathrm{mM} \mathrm{MgCl}, 1 \mathrm{mM}$ DTT, LPC cocktail [Leupeptin, Pepstatin, and Chymotrypsin, each at $10 \mu \mathrm{g} /$
$\mathrm{mL}])$ and subjected to nitrogen decompression using a Parr Instruments Cell Disruption Bomb. After centrifugation at $12,000 \mathrm{~g}$, the soluble fraction (Cytoplasmic) was removed and the remaining pellet resuspended in Buffer $\mathrm{B}(10 \mathrm{mM}$ Tris at $\mathrm{pH}$ 7.4, $420 \mathrm{mM} \mathrm{KCl}, 1.5 \mathrm{mM} \mathrm{MgCl} 2,25 \%$ Glycerol, $1 \mathrm{mM}$ DTT, LPC). Samples were spun again $(12,000 \mathrm{~g}$ for $10 \mathrm{~min})$, the supernatant was removed (Nuclear fraction), and the pellet was resuspended in Buffer B plus $0.1 \%$ Triton X-100. Samples were spun again, the supernatant was removed, and the pellet was resuspended in Buffer $\mathrm{B}+0.1 \%$ Triton $\mathrm{X}-100$ and sonicated $(20 \%$ duty cycle, $40 \mathrm{sec})$. After a final spin, the supernatant from each step was dialyzed into $20 \mathrm{mM}$ Tris (pH 7.4), $100 \mathrm{mM} \mathrm{KCl}$, 10\% Glycerol, 0.5 mM DTT, and 0.1 mM PMSF.

\section{Chromatography}

HeLa cell nuclear fractions were fractionated on a Q-Sepharose column and eluted with a $0.1-0.6 \mathrm{M} \mathrm{KCl}$ gradient. Pooled fractions containing Emil were separated on a Superose 6 gel filtration column (Amersham Biosciences) or a Superdex 200 gel filtration column (Amersham Biosciences). In addition, nuclear extracts were fractionated directly by Superose 6 gel filtration.

\section{Immunoprecipitation}

Affinity-purified anti-Emil antibody, mouse monoclonal IgG (Jackson Immunoresearch), anti-Emil rabbit polyclonal sera, anti-Cdc27 rabbit polyclonal sera, or preimmune sera were covalently coupled to protein A Affiprep beads (Bio-Rad). Beads were washed with IP Buffer (20 mM Tris at $\mathrm{pH} 7.4,100 \mathrm{mM}$ $\mathrm{KCl}, 0.1 \% \mathrm{NP}-40,10 \%$ Glycerol, $1 \mathrm{mM} \mathrm{MgCl} 2$, LPC) and incubated with HeLa cell nuclear fractions or pooled fractions from anion exchange $\left(4^{\circ} \mathrm{C}\right.$ for $\left.45 \mathrm{~min}\right)$. Immunoprecipitates were washed three times with IP Buffer, eluted with $2 \mathrm{M} \mathrm{MgCl}_{2}$ and separated by SDS-PAGE.

\section{Identification of SDS-PAGE bands by mass spectrometry}

Protein samples eluted from anti-Emil antibody beads were separated by SDS-PAGE and stained with Colloidal Blue Staining Kit (Invitrogen). Individual bands were excised from gel, reduced with DTT, and alkylated with acrylamide, resulting in propionamide-modified cysteines. Promega-modified trypsin was used for the proteolysis. Liquid chromatography-mass spectrometry/mass spectrometry (LC-MS/MS) was run with a peptide trap for online desalting, followed by a $0.150 \times 100-\mathrm{mm} \mathrm{C18}$ column, and elution directly into the ESI-ion trap mass spectrometer. The online Mascot MS/MS Ions Search from Matrix Science (http://www.matrixscience.com) was used for the database search.

\section{Plasmid and siRNA transfections}

HEK293T cells were transfected with pCS2 + myc or pCS2 + HA constructs and harvested as described previously (Hansen et al. 2004). siRNA oligoduplexes (Genentech) were transfected at $100 \mathrm{nM}$ final concentration using oligofectamine (Invitrogen). The target sequence of the Cdh1 oligoduplex is GCAACGATGTGTCTCCCTATT. The target sequence of the APC3 oligoduplex is GGAAATAGCCGAGAGGTAATT.

\section{Capture assay}

Immobilization of protein Recombinant proteins were coupled to CNBr-activated Sepharose 4B (Amersham Biosciences) in Coupling Buffer (0.1 $\mathrm{M} \mathrm{NaHCO}_{3}$ at $\mathrm{pH} 8.3,0.5 \mathrm{M}$ $\mathrm{NaCl}$ ) at a protein concentration of $0.1 \mu \mathrm{mol} / \mathrm{mL}$ medium $(16 \mathrm{~h}$ at $4^{\circ} \mathrm{C}$ ) and washed three times with Coupling Buffer, and re- 
maining active groups on medium were blocked with $0.1 \mathrm{M}$ Tris $(\mathrm{pH} 8.0)$ and $0.5 \mathrm{M} \mathrm{NaCl}\left(16 \mathrm{~h}\right.$ at $\left.4^{\circ} \mathrm{C}\right)$.

Binding assay CNBr-Sepharose containing $1 \mu \mathrm{mol}$ immobilized protein was incubated with $100 \mu \mathrm{L}$ of HeLa cell extract (10 $\mu \mathrm{g} / \mu \mathrm{L})$ in $10 \mathrm{mM}$ Hepes (pH 7.4), $100 \mathrm{mM} \mathrm{KCl}\left(2 \mathrm{~h}\right.$ at $\left.4^{\circ} \mathrm{C}\right)$. The samples were washed three times with $10 \mathrm{mM}$ Hepes (pH 7.4) and $250 \mathrm{mM} \mathrm{KCl}$ and bound proteins were resolved by SDSPAGE and immunoblotting. Reactions involving immobilized cyclin B were incubated at $20^{\circ} \mathrm{C}$.

Quantitation Levels of APC binding were measured by densiometry using ImageQuant program (GE Healthcare).

\section{Cdc20 depletion}

Stage VI oocytes were obtained and injected with $0.4 \mathrm{ng}$ each of two antisense oligonucleotides against Cdc20 (Taieb et al. 2001) in a maximum volume of $50 \mathrm{~nL}$. Oocytes were matured with 10 $\mu \mathrm{g} / \mathrm{mL}$ progesterone in OR2, and lysates were prepared as described (Hochegger et al. 2001).

\section{In vitro ubiquitination assay}

The assay was performed as described previously (Reimann et al. 2001b) using anti-Cdc27 immunoprecipitates from interphase Xenopus extract and hCdh1 as activator. Assays contained E1, E2, ubiquitin, ATP, and the ${ }^{35}$ S-labeled in vitro translated substrate (cyclin B for Fig. 5 and Supplementary Fig. 3A, Emil for Fig. 6.) Recombinant MBP fusion proteins were incubated for 30 min with activated anti-Cdc27 immunoprecipitates before addition of substrate. MBP fusion proteins were present throughout incubation with substrate for reactions presented in Figure 5. MBP fusions were removed before incubation with substrate for reactions presented in Supplementary Figure 3A.

\section{Acknowledgments}

We thank Jan-Michael Peters for anti-APC antibodies and Cdh1 $\Delta$ Cbox and $\Delta$ IR constructs; Hongtao Yu for anti-APC antibodies; Jeff Tung for assistance with the Cdc20 depletion experiment; Allis Chen, Andrew Guzzetta, and Evelyn Wang for performing mass spectrometry analyses; and Guowei Fang for critical reading of the manuscript. This work was supported by NIGMS grants RO1 GM60439 and RO1 GM54811 (to P.K.J.), NIGMS Medical Scientist Training Grant GM07365 (to J.J.M.), and the Tumor Biology Training Grant CA09151 (to M.K.S.).

\section{References}

Bashir, T., Dorrello, N.V., Amador, V., Guardavaccaro, D., and Pagano, M. 2004. Control of the SCF(Skp2-Cks1) ubiquitin ligase by the $\mathrm{APC} / \mathrm{C}(\mathrm{Cdh} 1)$ ubiquitin ligase. Nature 428: 190-193.

Burton, J.L. and Solomon, M.J. 2000. Hsllp, a Swelp inhibitor, is degraded via the anaphase-promoting complex. Mol. Cell. Biol. 20: 4614-4625.

Burton, J.L., Tsakraklides, V., and Solomon, M.J. 2005. Assembly of an APC-Cdh1-substrate complex is stimulated by engagement of a destruction box. Mol. Cell. 18: 533-542.

Carroll, C.W., Enquist-Newman, M., and Morgan, D.O. 2005. The APC subunit Docl promotes recognition of the substrate destruction box. Curr. Biol. 15: 11-18.

Castro, A., Bernis, C., Vigneron, S., Labbe, J.C., and Lorca, T. 2005. The anaphase-promoting complex: A key factor in the regulation of cell cycle. Oncogene 24: 314-325.

Davis, M., Hatzubai, A., Andersen, J.S., Ben-Shushan, E., Fisher, G.Z., Yaron, A., Bauskin, A., Mercurio, F., Mann, M., and Ben-Neriah, Y. 2002. Pseudosubstrate regulation of the $\operatorname{SCF}(\beta-\operatorname{TrCP})$ ubiquitin ligase by hnRNP-U. Genes \& Dev. 16: 439-451.

den Elzen, N. and Pines, J. 2001. Cyclin A is destroyed in prometaphase and can delay chromosome alignment and anaphase. J. Cell Biol. 153: 121-136.

Geley, S., Kramer, E., Gieffers, C., Gannon, J., Peters, J.M., and Hunt, T. 2001. Anaphase-promoting complex/cyclosome-dependent proteolysis of human cyclin A starts at the beginning of mitosis and is not subject to the spindle assembly checkpoint. J. Cell Biol. 153: 137-148.

Glotzer, M., Murray, A.W., and Kirschner, M.W. 1991. Cyclin is degraded by the ubiquitin pathway. Nature 349: 132-138.

Guardavaccaro, D., Kudo, Y., Boulaire, J., Barchi, M., Busino, L., Donzelli, M., Margottin-Goguet, F., Jackson, P.K., Yamasaki, L., and Pagano, M. 2003. Control of meiotic and mitotic progression by the $\mathrm{F}$ box protein $\beta$-Trcpl in vivo. Dev. Cell 4: 799-812.

Hansen, D.V., Loktev, A.V., Ban, K.H., and Jackson, P.K. 2004. Plk1 regulates activation of the anaphase promoting complex by phosphorylating and triggering SCF $\beta$ TrCP-dependent destruction of the APC Inhibitor Emil. Mol. Biol. Cell 15: 5623-5634.

Harper, J.W., Burton, J.L., and Solomon, M.J. 2002. The anaphase-promoting complex: It's not just for mitosis any more. Genes \& Dev. 16: 2179-2206.

Hilioti, Z., Chung, Y.S., Mochizuki, Y., Hardy, C.F., and CohenFix, O. 2001. The anaphase inhibitor Pds1 binds to the APC/ $\mathrm{C}$-associated protein Cdc20 in a destruction box-dependent manner. Curr. Biol. 11: 1347-1352.

Hochegger, H., Klotzbucher, A., Kirk, J., Howell, M., le Guellec, K., Fletcher, K., Duncan, T., Sohail, M., and Hunt, T. 2001. New B-type cyclin synthesis is required between meiosis I and II during Xenopus oocyte maturation. Development 128: 3795-3807.

Hoyt, M.A., Totis, L., and Roberts, B.T. 1991. S. cerevisiae genes required for cell cycle arrest in response to loss of microtubule function. Cell 66: 507-517.

Hsu, J.Y., Reimann, J.D., Sorensen, C.S., Lukas, J., and Jackson, P.K. 2002. E2F-dependent accumulation of hEmil regulates $S$ phase entry by inhibiting APC(Cdh1). Nat. Cell Biol. 4: 358-366.

Jackson, P.K. 2004. Linking tumor suppression, DNA damage and the anaphase-promoting complex. Trends Cell Biol. 14: 331-334.

King, R.W., Glotzer, M., and Kirschner, M.W. 1996. Mutagenic analysis of the destruction signal of mitotic cyclins and structural characterization of ubiquitinated intermediates. Mol. Biol. Cell 7: 1343-1357.

Kraft, C., Vodermaier, H.C., Maurer-Stroh, S., Eisenhaber, F., and Peters, J.M. 2005. The WD40 propeller domain of Cdh1 functions as a destruction box receptor for APC/C substrates. Mol. Cell 18: 543-553.

Li, R. and Murray, A.W. 1991. Feedback control of mitosis in budding yeast. Cell 66: 519-531.

Margottin-Goguet, F., Hsu, J.Y., Loktev, A., Hsieh, H.M., Reimann, J.D., and Jackson, P.K. 2003. Prophase destruction of Emil by the SCF( $\beta$ TrCP/Slimb) ubiquitin ligase activates the anaphase promoting complex to allow progression beyond prometaphase. Dev. Cell 4: 813-826.

Meraldi, P., Draviam, V.M., and Sorger, P.K. 2004. Timing and checkpoints in the regulation of mitotic progression. Dev. Cell 7: 45-60. 
Miller et al.

Moshe, Y., Boulaire, J., Pagano, M., and Hershko, A. 2004. Role of Polo-like kinase in the degradation of early mitotic inhibitor 1, a regulator of the anaphase promoting complex/cyclosome. Proc. Natl. Acad. Sci. 101: 7937-7942.

Nasmyth, K. 2005. How do so few control so many? Cell 120: 739-746.

Passmore, L.A., McCormack, E.A., Au, S.W., Paul, A., Willison, K.R., Harper, J.W., and Barford, D. 2003. Doc1 mediates the activity of the anaphase-promoting complex by contributing to substrate recognition. EMBO J. 22: 786-796.

Pfleger, C.M. and Kirschner, M.W. 2000. The KEN box: An APC recognition signal distinct from the $\mathrm{D}$ box targeted by Cdh1. Genes \& Dev. 14: 655-665.

Pfleger, C.M., Lee, E., and Kirschner, M.W. 2001. Substrate recognition by the Cdc2 0 and Cdh1 components of the anaphase-promoting complex. Genes \& Dev. 15: 2396-2407.

Reimann, J.D., Freed, E., Hsu, J.Y., Kramer, E.R., Peters, J.M., and Jackson, P.K. 2001a. Emil is a mitotic regulator that interacts with Cdc20 and inhibits the anaphase promoting complex. Cell 105: 645-655.

Reimann, J.D., Gardner, B.E., Margottin-Goguet, F., and Jackson, P.K. 2001b. Emil regulates the anaphase-promoting complex by a different mechanism than Mad2 proteins. Genes \& Dev. 15: 3278-3285.

Rieder, C.L. and Maiato, H. 2004. Stuck in division or passing through: What happens when cells cannot satisfy the spindle assembly checkpoint. Dev. Cell 7: 637-651.

Russo, A.A., Jeffrey, P.D., Patten, A.K., Massague, J., and Pavletich, N.P. 1996. Crystal structure of the p27Kip1 cyclindependent-kinase inhibitor bound to the cyclin A-Cdk2 complex. Nature 382: 325-331.

Schmidt, A., Duncan, P.I., Rauh, N.R., Sauer, G., Fry, A.M., Nigg, E.A., and Mayer, T.U. 2005. Xenopus polo-like kinase Plx1 regulates XErp1, a novel inhibitor of APC/C activity. Genes \& Dev. 19: 502-513.

Schwab, M., Lutum, A.S., and Seufert, W. 1997. Yeast Hct1 is a regulator of Clb2 cyclin proteolysis. Cell 90: 683-693.

Song, M.S., Song, S.J., Ayad, N.G., Chang, J.S., Lee, J.H., Hong, H.K., Lee, H., Choi, N., Kim, J., Kim, H., et al. 2004. The tumour suppressor RASSF1A regulates mitosis by inhibiting the APC-Cdc20 complex. Nat. Cell Biol. 6: 129-137.

Taieb, F.E., Gross, S.D., Lewellyn, A.L., and Maller, J.L. 2001. Activation of the anaphase-promoting complex and degradation of cyclin B is not required for progression from Meiosis I to II in Xenopus oocytes. Curr. Biol. 11: 508-513.

Tung, J.J., Hansen, D.V., Ban, K.H., Loktev, A.V., Summers, M.K., Adler III, J.R., and Jackson, P.K. 2005. A role for the anaphase-promoting complex inhibitor Emi2/XErp1, a homolog of early mitotic inhibitor 1 , in cytostatic factor arrest of Xenopus eggs. Proc. Natl. Acad. Sci. 102: 4318-4323.

van der Reijden, B.A., Erpelinck-Verschueren, C.A., Lowenberg, B., and Jansen, J.H. 1999. TRIADs: A new class of proteins with a novel cysteine-rich signature. Protein Sci. 8: 15571561.

Visintin, R., Prinz, S., and Amon, A. 1997. CDC20 and CDH1: A family of substrate-specific activators of APC-dependent proteolysis. Science 278: 460-463.

Yamano, H., Gannon, J., Mahbubani, H., and Hunt, T. 2004. Cell cycle-regulated recognition of the destruction box of cyclin B by the APC/C in Xenopus egg extracts. Mol. Cell 13: 137-147.

Yu, H., Peters, J.M., King, R.W., Page, A.M., Hieter, P., and Kirschner, M.W. 1998. Identification of a cullin homology region in a subunit of the anaphase-promoting complex. Science 279: 1219-1222.

Zachariae, W., Schwab, M., Nasmyth, K., and Seufert, W. 1998.
Control of cyclin ubiquitination by CDK-regulated binding of Hct1 to the anaphase promoting complex. Science 282: $1721-1724$. 


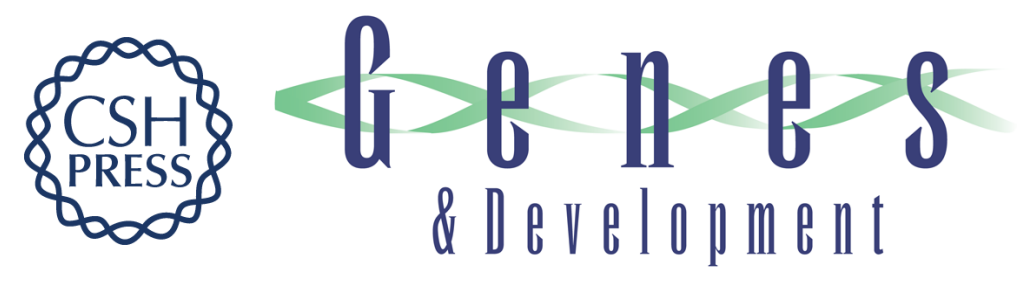

\section{Emi1 stably binds and inhibits the anaphase-promoting complex/cyclosome as a pseudosubstrate inhibitor}

Julie J. Miller, Matthew K. Summers, David V. Hansen, et al.

Genes Dev. 2006, 20:

Access the most recent version at doi:10.1101/gad.1454006

\section{Supplemental http://genesdev.cshlp.org/content/suppl/2006/09/05/gad.1454006.DC1 Material}

References This article cites 41 articles, 18 of which can be accessed free at: http://genesdev.cshlp.org/content/20/17/2410.full.html\#ref-list-1

\section{License}

Email Alerting

Receive free email alerts when new articles cite this article - sign up in the box at the top Service 